\title{
VISCOSITY APPROXIMATION METHODS FOR EQUILIBRIUM PROBLEMS AND FIXED POINT PROBLEMS OF NONEXPANSIVE MAPPINGS AND INVERSE-STRONGLY MONOTONE MAPPINGS*
}

\author{
SHENGHUA WANG ${ }^{\dagger}$, HAIYUN ZHOU ${ }^{\ddagger}$, AND JIANMIN SONG§
}

Abstract. In this paper, we introduce an iterative scheme by viscosity approximation method for obtaining a common element of the set of solutions of an equilibrium problem and the set of fixed points of a nonexpansive mapping and the set of solutions of the variational inequality for an inverse-strongly monotone mapping in a Hilbert space. We obtain a strong convergence which improves and extends S. Takahashi and W. Takahashi's result [S. Takahashi, W. Takahashi, Viscosity approximation methods for equilibrium problems and fixed point problems in Hilbert spaces, J. Math. Anal. Appl. 331 (2007) 506-515].

Key words. Viscosity approximation method; Equilibrium problem; Inverse-strongly monotone mapping; Nonexpansive mapping; Variational inequality.

AMS subject classifications. 47H05, 47H10, 47H17

1. Introduction and Preliminaries. Let $C$ be a nonempty closed convex subset of a real Hilbert $H$. Let $f$ be a mapping from $C$ into itself. Then, $f$ is called a contraction on $C$ if there exists a constant $\kappa \in(0,1)$ such that

$$
\|f(x)-f(y)\| \leq \kappa\|x-y\|, \text { for all } x, y \in C .
$$

We denote the set of all contractions on $C$ by $\Pi_{C}$. Note that $f$ has a unique fixed point in $C$.

Let $S$ be a mapping from $C$ into itself, then $S$ is called nonexpansive if

$$
\|S x-S y\| \leq\|x-y\|
$$

for all $x, y \in C$. In this paper, we denote the set of fixed points of $S$ by $F(S)$.

Let $F$ be a bifunction of $C \times C$ into $\mathbf{R}$, where $\mathbf{R}$ is the set of real numbers. The equilibrium problem for $F: C \times C \rightarrow \mathbf{R}$ is to find $x \in C$ such that

$$
F(x, y) \geq 0 \text { for all } y \in C \text {. }
$$

For solving above equilibrium problem, assume that $F$ satisfies the following conditions:

(A1) $F(x, x)=0$ for all $x \in C$;

(A2) $F$ is monotone, i.e., $F(x, y)+F(y, x) \leq 0$ for all $x, y \in C$;

(A3) for each $x, y, z \in C, \lim _{t \downarrow 0} F(t z+(1-t) x, y) \leq F(x, y)$;

(A4) for each $x \in C, y \mapsto F(x, y)$ is convex and lower semicontinuous.

The set of solution of the above equilibrium problem is denoted by $\operatorname{EP}(F)$. The following lemmas were given in [2] and [5], respectively.

\footnotetext{
* Received October 2, 2007; accepted for publication July 15, 2008.

${ }^{\dagger}$ Corresponding author.

${ }^{\ddagger}$ Institute of Nonlinear Anal., North China Electric Power University, Baoding 071003, P.R. China (sheng-huawang@hotmail.com; witman66@yahoo.com.cn).

$\S$ School Mathematics and Science, Shijiazhuang University of Economics, Shijiazhuang, 050031, P. R. China (jianminsong@yahoo.cn).
} 
LemMa 1.1 ([2]). Let $C$ be a nonempty closed convex subset of $H$ and let $F$ be a bifunction of $C \times C$ into $\mathbf{R}$ satisfies (A1) - (A4). Let $r>0$ and $x \in H$. Then, there exists $z \in C$ such that

$$
F(z, y)+\frac{1}{r}\langle y-z, z-x\rangle \geq 0 \text { for all } y \in C
$$

Lemma $1.2([5])$. Assume that $F: C \times C \rightarrow \mathbf{R}$ satisfies $(\mathrm{A} 1)-(\mathrm{A} 4)$. For $r>0$ and $x \in H$, define a mapping $T_{r}: H \rightarrow C$ as follows:

$$
T_{r}(x)=\left\{z \in C: F(z, y)+\frac{1}{r}\langle y-z, z-x\rangle \geq 0, \forall y \in C\right\}
$$

for all $x \in H$. Then, the following hold:

(1) $T_{r}$ is single-valued;

(2) $T_{r}$ is firmly nonexpansive, i.e., for any $x, y \in H$,

$$
\left\|T_{r} x-T_{r} y\right\|^{2} \leq\left\langle T_{r} x-T_{r} y, x-y\right\rangle
$$

(3) $F\left(T_{r}\right)=E P(F)$;

(4) $E P(F)$ is closed and convex.

Lemma 1.2 shows that for each given $x \in H$, there exists a unique $T_{r}(x) \in C$. However, it is very hard to find such a $z \in C$ such that

$$
F(z, y)+\frac{1}{r}\langle y-z, z-x\rangle \geq 0
$$

for all $y \in C$, that is, for a given $x \in H$, it is very hard to compute $T_{r}(x)$. In [5], Combettes and Hirstoaga gave an iterative algorithm to compute $T_{r}(x)$ for a given $x \in H$. On this problem, the interested readers may refer to [5]. Here, we give a simple example to compute $T_{r}(x)$ in a Euclidean space. Put $H=R^{2}$ and $C=\{x \in H:\|x\| \leq 1\}$. Let $F(x, y)=\|y\|^{2}-\|x\|^{2}$ for all $x, y \in C$. Obviously, the bifunction $F$ satisfies the conditions $\mathrm{A}(1)-\mathrm{A}(4)$. Taking $r=1$, for given $x=0$, we compute

$$
T_{1}(0)=\{z \in C: F(z, y)+\langle y-z, z\rangle \geq 0, \forall y \in C\}
$$

Note that $F(z, y)+\langle y-z, z\rangle \geq 0$ is equivalent to the inequality

$$
2\|z\|^{2} \leq\|y\|^{2}+\langle y-z, z\rangle
$$

and observe that $z=0$ satisfies the above inequality for all $y \in C$. Since $T_{1}$ is single-value from Lemma 1.2 , we know that $T_{1}(0)=0$.

Let $A$ be a mapping from $C$ into $H$, then $A$ is called monotone if

$$
\langle x-y, A x-A y\rangle \geq 0
$$

for all $x, y \in C$. However, $A$ is called an $\alpha$-inverse-strongly monotone mapping if there exists a positive real number $\alpha$ such that

$$
\langle x-y, A x-A y\rangle \geq \alpha\|A x-A y\|^{2}
$$


for all $x, y \in C$. Let $I$ denote the identity mapping of $H$, then for all $x, y \in C$ and $\lambda>0$, one has $[6]$

$$
\|(I-\lambda A) x-(I-\lambda A) y\|^{2} \leq\|x-y\|^{2}+\lambda(\lambda-2 \alpha)\|A x-A y\|^{2} .
$$

Hence, if $\lambda \in(0,2 \alpha]$, then $I-\lambda A$ is a nonexpansive mapping of $C$ into $H$.

If there exists $u \in C$ such that

$$
\langle v-u, A u\rangle \geq 0
$$

for all $v \in C$, then $u$ is called the solution of this variational inequality. The set of all solutions of the variational inequality is denoted by $\operatorname{VI}(C, A)$.

For every point $x \in H$, there exists a unique nearest point in $C$, denoted by $P_{C} x$, such that

$$
\left\|x-P_{C} x\right\| \leq\|x-y\|
$$

for all $y \in C$. $P_{C}$ is called the metric projection of $H$ onto $C$. It is well known that $P_{C}$ is a nonexpansive mapping of $H$ onto $C$ and satisfies

$$
\left\langle x-y, P_{C} x-P_{C} y\right\rangle \geq\left\|P_{C} x-P_{C} y\right\|^{2}
$$

for all $x, y \in H$. Moreover, for every $x \in H$, one has

$$
\left\langle x-P_{C} x, P_{C} x-y\right\rangle \geq 0
$$

for all $y \in C$, which implies that

$$
u \in V I(C, A) \Leftrightarrow u=P_{C}(u-\lambda A u), \quad \forall \lambda>0 .
$$

Recently, for obtaining an element of $F(S) \cap V I(C, A)$, Iiduka and Takahashi [6] introduced the following iterative algorithm: $x_{1}=x \in C$ and

$$
x_{n+1}=\alpha_{n} x+\left(1-\alpha_{n}\right) S P_{C}\left(x_{n}-\lambda_{n} A x_{n}\right), \quad n \geq 1
$$

and obtained a strong convergence theorem. On the other hand, for finding the element of $F(S) \cap E P(F)$, Takahashi and Takahashi [9] introduced the following algorithm: $x_{1} \in H$ and

$$
\left\{\begin{array}{l}
F\left(u_{n}, y\right)+\frac{1}{r_{n}}\left\langle y-u_{n}, u_{n}-x_{n}\right\rangle \geq 0, \quad \forall y \in C, \\
x_{n+1}=\alpha_{n} f\left(x_{n}\right)+\left(1-\alpha_{n}\right) S u_{n}
\end{array}\right.
$$

for all $n \geq 1$. They proved that $\left\{x_{n}\right\}$ and $\left\{u_{n}\right\}$ converge strongly to $z \in F(S) \cap E P(F)$, where $z=P_{F(S) \cap E P(F)} f(z)$ if $\left\{\alpha_{n}\right\} \subset[0,1]$ and $\left\{r_{n}\right\} \subset(0, \infty)$ satisfy some certain conditions.

In literatures, many iterative methods for finding the common point of $F(S)$ and $V I(C, A)$ or $E P(F)$ have been proposed and studied widely. For example, see $[10,7,4]$. However, the algorithm for approximating the element of the intersection of $F(S), V I(C, A)$ and $E P(F)$ have not been found in literatures. In order to obtain the common point of $F(S), V I(C, A)$ and $E P(F)$, we in this paper introduce an iterative scheme by the viscosity approximation method to find an element $z \in F(S) \cap$ $V I(C, A) \cap E P(F)$. Our result improves and extends S. Takahashi and W. Takahashi's 
result [9]. Using this result, we obtain two corollaries which are connected with Combettes and Hirstoaga's result [5].

The following lemmas are useful.

LEMMA $1.3([8])$. Let $\left\{x_{n}\right\}$ and $\left\{w_{n}\right\}$ be bounded sequences in a Banach space $X$ and let $\left\{\beta_{n}\right\}$ be a sequence in $[0,1]$ with $0<\liminf _{n \rightarrow \infty} \beta_{n}<\limsup _{n \rightarrow \infty} \beta_{n}<1$. Suppose

$$
x_{n+1}=\beta_{n} x_{n}+\left(1-\beta_{n}\right) w_{n}
$$

for all integers $n \geq 0$ and $\lim \sup _{n \rightarrow \infty}\left(\left\|w_{n+1}-w_{n}\right\|-\left\|x_{n+1}-x_{n}\right\|\right) \leq 0$. Then,

$$
\lim _{n \rightarrow \infty}\left\|w_{n}-x_{n}\right\|=0 \text {. }
$$

LEMMA $1.4([11])$. Let $\left\{a_{n}\right\}$ be a non-negative real number sequence satisfying

$$
a_{n+1} \leq\left(1-\alpha_{n}\right) a_{n}+o\left(\alpha_{n}\right), \quad n=0,1,2, \cdots,
$$

where $\left\{\alpha_{n}\right\} \subset(0,1)$ is a real number sequence. If $\sum_{n=0}^{\infty} \alpha_{n}=\infty$, then $\lim _{n \rightarrow \infty} a_{n}=0$.

\section{Main result.}

THEOREM 2.1. Let $C$ be a nonempty closed convex subset of a real Hilbert space $H$. Let $F$ be a bifunction from $C \times C$ to $\mathbf{R}$ satisfying (A1) - (A4) and $f$ be a contraction with coefficient $\kappa(0<\kappa<1)$ from $C$ into itself. Let $A$ be an $\alpha$-inverse-strongly monotone mapping of $C$ into $H$ and let $S$ be a nonexpansive mapping of $C$ into itself such that $F(S) \cap E P(F) \cap V I(C, A) \neq \emptyset$. Suppose $x_{1}=x \in C$ and $\left\{x_{n}\right\},\left\{u_{n}\right\}$ are sequences generated by

$$
\left\{\begin{array}{l}
F\left(u_{n}, y\right)+\frac{1}{r_{n}}\left\langle y-u_{n}, u_{n}-x_{n}\right\rangle \geq 0, \quad \forall y \in C, \\
x_{n+1}=\alpha_{n} f\left(x_{n}\right)+\beta_{n} x_{n}+\gamma_{n}\left(\mu S u_{n}+(1-\mu) P_{C}\left(u_{n}-\lambda_{n} A u_{n}\right)\right),
\end{array}\right.
$$

for every $n=1,2, \cdots$, where $\mu \in[0,1],\left\{r_{n}\right\} \subset(0, \infty),\left\{\lambda_{n}\right\} \subset[a, b]$ with $0<a<b<$ $2 \alpha$ and $\left\{\alpha_{n}\right\},\left\{\beta_{n}\right\}$ and $\left\{\gamma_{n}\right\}$ are sequences in $[0,1]$ and satisfy $\alpha_{n}+\beta_{n}+\gamma_{n}=1$ for every $n=1,2, \cdots$. If $\left\{\alpha_{n}\right\},\left\{\beta_{n}\right\},\left\{\lambda_{n}\right\}$ and $\left\{r_{n}\right\}$ are chosen so that

$$
\begin{aligned}
& \lim _{n \rightarrow \infty} \alpha_{n}=0, \quad \sum_{n=1}^{\infty} \alpha_{n}=\infty, \quad 0<\liminf _{n \rightarrow \infty} \beta_{n}<\limsup _{n \rightarrow \infty} \beta_{n}<1, \\
& \lim _{n \rightarrow \infty}\left|\lambda_{n+1}-\lambda_{n}\right|=0, \quad \liminf _{n \rightarrow \infty} r_{n}>0 \text { and } \lim _{n \rightarrow \infty}\left|r_{n+1}-r_{n}\right|=0,
\end{aligned}
$$

then $\left\{x_{n}\right\}$ and $\left\{u_{n}\right\}$ converge strongly to the same point $z \in F(S) \cap E P(F) \cap V I(C, A)$, where $z=P_{F(S) \cap E P(F) \cap V I(C, A)} f(z)$.

Proof. We proceed with the following steps.

Step 1. $\left\{x_{n}\right\}$ is bounded.

Let $Q=P_{F(S) \cap E P(F) \cap V I(C, A)}$. Then $Q f$ is a contraction of $C$ into itself. In fact, for all $x, y \in C$, we have $\|Q f(x)-Q f(y)\| \leq\|f(x)-f(y)\| \leq \kappa\|x-y\|$. So, $Q f$ is a contraction of $C$ into itself. Since $C$ is complete, there exists a unique element $z \in C$ such that $z=Q f(z)$. 
Let $v \in F(S) \cap E P(F) \cap V I(C, A)$. Since $u_{n}=T_{r_{n}} x_{n}$, we have

$$
\left\|u_{n}-v\right\|=\left\|T_{r_{n}} x_{n}-T_{r_{n}} v\right\| \leq\left\|x_{n}-v\right\|
$$

for every $n=1,2, \cdots$.

Let $z_{n}=\mu S u_{n}+(1-\mu) t_{n}$, where $t_{n}=P_{C}\left(u_{n}-\lambda_{n} A u_{n}\right)$, for every $n=1,2, \cdots$. Then, we have

$$
\begin{aligned}
\left\|t_{n}-v\right\| & =\left\|P_{C}\left(u_{n}-\lambda_{n} A u_{n}\right)-P_{C}\left(v-\lambda_{n} A v\right)\right\| \\
& \leq\left\|u_{n}-\lambda_{n} A u_{n}-\left(v-\lambda_{n} A v\right)\right\| \\
& \leq\left\|u_{n}-v\right\| \\
& \leq\left\|x_{n}-v\right\|
\end{aligned}
$$

and

$$
\begin{aligned}
\left\|z_{n}-v\right\| & =\left\|\mu\left(S u_{n}-v\right)+(1-\mu)\left(t_{n}-v\right)\right\| \\
& \leq \mu\left\|u_{n}-v\right\|+(1-\mu)\left\|t_{n}-v\right\| \\
& \leq \mu\left\|x_{n}-v\right\|+(1-\mu)\left\|x_{n}-v\right\| \\
& =\left\|x_{n}-v\right\|,
\end{aligned}
$$

for every $n=1,2, \cdots$.

Put $M=\max \left\{\left\|x_{1}-v\right\|, \frac{1}{1-\kappa}\|f(v)-v\|\right\}$. Suppose $\left\|x_{n}-v\right\| \leq M$. Then we have

$$
\begin{array}{ll} 
& \left\|x_{n+1}-v\right\| \\
=\quad & \left\|\alpha_{n}\left(f\left(x_{n}\right)-v\right)+\beta_{n}\left(x_{n}-v\right)+\gamma_{n}\left(z_{n}-v\right)\right\| \\
\leq & \alpha_{n}\left\|f\left(x_{n}\right)-v\right\|+\beta_{n}\left\|x_{n}-v\right\|+\gamma_{n}\left\|x_{n}-v\right\| \\
\leq \quad & \alpha_{n}\left\|f\left(x_{n}\right)-f(v)\right\|+\alpha_{n}\|f(v)-v\|+\left(1-\alpha_{n}\right)\left\|x_{n}-v\right\| \\
\leq \quad & \left(1-\alpha_{n}(1-\kappa)\right)\left\|x_{n}-v\right\|+\alpha_{n}(1-\kappa) \frac{1}{1-\kappa}\|f(v)-v\| \\
\leq & \left(1-\alpha_{n}(1-\kappa)\right) M+\alpha_{n}(1-\kappa) M=M .
\end{array}
$$

Noting $\left\|x_{1}-v\right\| \leq M$, by mathematical induction, we have $\left\|x_{n}-v\right\| \leq M$ for all $n \in N$. Hence, $\left\{x_{n}\right\}$ is bounded and $\left\{u_{n}\right\},\left\{f\left(x_{n}\right)\right\},\left\{z_{n}\right\}$ and $\left\{A u_{n}\right\}$ are all bounded.

Step 2. $\lim _{n \rightarrow \infty}\left\|x_{n+1}-x_{n}\right\|=0$.

Since $u_{n}=T_{r_{n}} x_{n}$ and $u_{n+1}=T_{r_{n+1}} x_{n+1}$, we have

$$
F\left(u_{n}, y\right)+\frac{1}{r_{n}}\left\langle y-u_{n}, u_{n}-x_{n}\right\rangle \geq 0 \text { for all } y \in C
$$

and

$$
F\left(u_{n+1}, y\right)+\frac{1}{r_{n+1}}\left\langle y-u_{n+1}, u_{n+1}-x_{n+1}\right\rangle \geq 0 \text { for all } y \in C
$$

Putting $y=u_{n+1}$ in (2.1) and $y=u_{n}$ in (2.2), we have

$$
F\left(u_{n}, u_{n+1}\right)+\frac{1}{r_{n}}\left\langle u_{n+1}-u_{n}, u_{n}-x_{n}\right\rangle \geq 0
$$

and

$$
F\left(u_{n+1}, u_{n}\right)+\frac{1}{r_{n+1}}\left\langle u_{n}-u_{n+1}, u_{n+1}-x_{n+1}\right\rangle \geq 0 .
$$


Therefore, from (A2) we have

$$
\left\langle u_{n+1}-u_{n}, \frac{u_{n}-x_{n}}{r_{n}}-\frac{u_{n+1}-x_{n+1}}{r_{n+1}}\right\rangle \geq 0
$$

and hence

$$
\left\langle u_{n+1}-u_{n}, u_{n}-u_{n+1}+u_{n+1}-x_{n}-\frac{r_{n}}{r_{n+1}}\left(u_{n+1}-x_{n+1}\right)\right\rangle \geq 0 .
$$

Since $\left\{r_{n}\right\} \subset(0, \infty)$, there exists a real number $b$ such that $r_{n}>b>0$ for every $n=1,2, \cdots$. Then, we have

$$
\begin{aligned}
\left\|u_{n+1}-u_{n}\right\|^{2} & \leq\left\langle u_{n+1}-u_{n}, x_{n+1}-x_{n}+\left(1-\frac{r_{n}}{r_{n+1}}\right)\left(u_{n+1}-x_{n+1}\right)\right\rangle \\
& \leq\left\|u_{n+1}-u_{n}\right\|\left\{\left\|x_{n+1}-x_{n}\right\|+\left|1-\frac{r_{n}}{r_{n+1}}\right|\left\|u_{n+1}-x_{n+1}\right\|\right\}
\end{aligned}
$$

and hence

$$
\begin{aligned}
\left\|u_{n+1}-u_{n}\right\| & \leq\left\|x_{n+1}-x_{n}\right\|+\frac{1}{r_{n+1}}\left|r_{n+1}-r_{n}\right|\left\|u_{n+1}-x_{n+1}\right\| \\
& \leq\left\|x_{n+1}-x_{n}\right\|+\frac{1}{b}\left|r_{n+1}-r_{n}\right| L
\end{aligned}
$$

where $L=\sup \left\{\left\|u_{n}-x_{n}\right\|: n=1,2, \cdots\right\}$.

Putting $w_{n}=\frac{x_{n+1}-\beta_{n} x_{n}}{1-\beta_{n}}$, for every $n=1,2, \cdots$, then we obtain

$$
\begin{aligned}
& w_{n+1}-w_{n} \\
= & \frac{\alpha_{n+1} f\left(x_{n+1}\right)+\gamma_{n+1} z_{n+1}}{1-\beta_{n+1}}-\frac{\alpha_{n} f\left(x_{n}\right)+\gamma_{n} z_{n}}{1-\beta_{n}} \\
= & \frac{\alpha_{n+1}\left(f\left(x_{n+1}-f\left(x_{n}\right)\right)\right.}{1-\beta_{n+1}}+\frac{\alpha_{n+1} f\left(x_{n}\right)}{1-\beta_{n+1}}+\frac{\gamma_{n+1}\left(z_{n+1}-z_{n}\right)}{1-\beta_{n+1}}+\frac{\gamma_{n+1} z_{n}}{1-\beta_{n+1}}-\frac{\alpha_{n} f\left(x_{n}\right)+\gamma_{n} z_{n}}{1-\beta_{n}} \\
= & \frac{\alpha_{n+1}\left(f\left(x_{n+1}\right)-f\left(x_{n}\right)\right)}{1-\beta_{n+1}}+\frac{\alpha_{n+1} f\left(x_{n}\right)}{1-\beta_{n+1}}+\frac{\gamma_{n+1}\left(z_{n+1}-z_{n}\right)}{1-\beta_{n+1}}+z_{n}-\frac{\alpha_{n+1} z_{n}}{1-\beta_{n+1}} \\
& -\frac{\alpha_{n} f\left(x_{n}\right)}{1-\beta_{n}}-z_{n}+\frac{\alpha_{n} z_{n}}{1-\beta_{n}} \\
= & \frac{\alpha_{n+1}\left(f\left(x_{n+1}\right)-f\left(x_{n}\right)\right)}{1-\beta_{n+1}}+\frac{\gamma_{n+1}\left(z_{n+1}-z_{n}\right)}{1-\beta_{n+1}}+\frac{\alpha_{n+1}\left(f\left(x_{n}\right)-z_{n}\right)}{1-\beta_{n+1}}+\frac{\alpha_{n}\left(z_{n}-f\left(x_{n}\right)\right.}{1-\beta_{n}} .
\end{aligned}
$$

By using (2.3) we have

$$
\begin{aligned}
\left\|t_{n+1}-t_{n}\right\| & =\| P_{C}\left(u_{n+1}-\lambda_{n+1} A u_{n+1}\right)-P_{C}\left(u_{n}-\lambda_{n} A u_{n}\right) \\
& \leq\left\|u_{n+1}-\lambda_{n+1} A u_{n+1}-\left(u_{n}-\lambda_{n} A u_{n}\right)\right\| \\
& =\left\|u_{n+1}-\lambda_{n+1} A u_{n+1}-\left(u_{n}-\lambda_{n+1} A u_{n}\right)+\left(\lambda_{n}-\lambda_{n+1}\right) A u_{n}\right\| \\
& \leq\left\|u_{n+1}-\lambda_{n+1} A u_{n+1}-\left(u_{n}-\lambda_{n+1} A u_{n}\right)\right\|+\left|\lambda_{n}-\lambda_{n+1}\right|\left\|A u_{n}\right\| \\
& \leq\left\|u_{n+1}-u_{n}\right\|+\left|\lambda_{n}-\lambda_{n+1}\right|\left\|A u_{n}\right\| \\
& \leq\left\|x_{n+1}-x_{n}\right\|+\frac{1}{b}\left|r_{n+1}-r_{n}\right| L+\left|\lambda_{n}-\lambda_{n+1}\right|\left\|A u_{n}\right\|,
\end{aligned}
$$

for every $n=1,2, \cdots$. Therefore, we obtain

$$
\begin{aligned}
& \left\|z_{n+1}-z_{n}\right\| \\
= & \left\|\mu\left(S u_{n+1}-S u_{n}\right)+(1-\mu)\left(t_{n+1}-t_{n}\right)\right\| \\
\leq & \mu\left\|u_{n+1}-u_{n}\right\|+(1-\mu)\left\|t_{n+1}-t_{n}\right\| \\
\leq & \mu\left(\left\|x_{n+1}-x_{n}\right\|+\frac{1}{b}\left|r_{n+1}-r_{n}\right| L\right)+(1-\mu)\left(\left\|x_{n+1}-x_{n}\right\|+\frac{1}{b}\left|r_{n+1}-r_{n}\right| L\right) \\
& +(1-\mu)\left|\lambda_{n}-\lambda_{n+1}\right|\left\|A u_{n}\right\| \\
\leq & \left\|x_{n+1}-x_{n}\right\|+\frac{1}{b}\left|r_{n+1}-r_{n}\right| L+\left|\lambda_{n}-\lambda_{n+1}\right|\left\|A u_{n}\right\|
\end{aligned}
$$


and

$$
\begin{aligned}
& \left\|w_{n+1}-w_{n}\right\|-\left\|x_{n+1}-x_{n}\right\| \\
\leq & \frac{\alpha_{n+1}\left(\left\|f\left(x_{n+1}\right)\right\|+\left\|f\left(x_{n}\right)\right\|\right)}{1-\beta_{n+1}}+\frac{\alpha_{n+1}\left(\left\|f\left(x_{n}\right)\right\|+\left\|z_{n}\right\|\right)}{1-\beta_{n+1}}+\frac{\alpha_{n}\left(\left\|f\left(x_{n}\right)\right\|+\left\|z_{n}\right\|\right)}{1-\beta_{n}} \\
& +\frac{\gamma_{n+1}\left\|x_{n+1}-x_{n}\right\|}{1-\beta_{n+1}}+\frac{\gamma_{n+1} \frac{1}{b}\left|r_{n+1}-r_{n}\right| L}{1-\beta_{n+1}}+\frac{\gamma_{n+1}\left|\lambda_{n}-\lambda_{n+1}\right|\left\|A u_{n}\right\|}{1-\beta_{n+1}} \\
& -\left\|x_{n+1}-x_{n}\right\| \\
\leq & \frac{\alpha_{n+1}\left(\left\|f\left(x_{n+1}\right)\right\|+\left\|f\left(x_{n}\right)\right\|\right)}{1-\beta_{n+1}}+\frac{\alpha_{n+1}\left(\left\|f\left(x_{n}\right)\right\|+\left\|z_{n}\right\|\right)}{1-\beta_{n+1}}+\frac{\alpha_{n}\left(\left\|f\left(x_{n}\right)\right\|+\left\|z_{n}\right\|\right)}{1-\beta_{n}} \\
& +\frac{\gamma_{n+1} \frac{1}{b}\left|r_{n+1}-r_{n}\right| L}{1-\beta_{n+1}}+\frac{\gamma_{n+1}\left|\lambda_{n}-\lambda_{n+1}\right|\left\|A u_{n}\right\|}{1-\beta_{n+1}},
\end{aligned}
$$

for every $n=1,2, \cdots$. Since $\left\{f\left(x_{n}\right)\right\},\left\{z_{n}\right\}$ and $\left\{A u_{n}\right\}$ are bounded, and $\lim _{n \rightarrow \infty} \alpha_{n}=$ $0, \lim _{n \rightarrow \infty}\left\|r_{n+1}-r_{n}\right\|=0$ and $\lim _{n \rightarrow \infty}\left\|\lambda_{n+1}-\lambda_{n}\right\|=0$, we have

$$
\limsup _{n \rightarrow \infty}\left(\left\|w_{n+1}-w_{n}\right\|-\left\|x_{n+1}-x_{n}\right\|\right) \leq 0 .
$$

According to Lemma 1.3, we obtain $\lim _{n \rightarrow \infty}\left\|w_{n}-x_{n}\right\|=0$, i.e., $\lim _{n \rightarrow \infty} \frac{1}{1-\beta_{n}} \| x_{n+1}-$ $x_{n} \|=0$. Noting that $0<\liminf _{n \rightarrow \infty} \beta_{n}<\limsup _{n \rightarrow \infty} \beta_{n}<1$, we have

$$
\lim _{n \rightarrow \infty}\left\|x_{n+1}-x_{n}\right\|=0
$$

Step 3. $\lim _{n \rightarrow \infty}\left\|x_{n}-u_{n}\right\|=0$.

First we prove that $\lim _{n \rightarrow \infty}\left\|A u_{n}-A v\right\|=0$ and $\lim _{n \rightarrow \infty}\left\|u_{n}-t_{n}\right\|=0$.

By using (1.1) we have

$$
\begin{aligned}
\left\|t_{n}-v\right\|^{2} & =\left\|P_{C}\left(u_{n}-\lambda_{n} A u_{n}\right)-P_{C}\left(v-\lambda_{n} A v\right)\right\|^{2} \\
& \leq\left\|u_{n}-\lambda_{n} A u_{n}-\left(v-\lambda_{n} A v\right)\right\|^{2} \\
& \leq\left\|u_{n}-v\right\|^{2}+\lambda_{n}\left(\lambda_{n}-2 \alpha\right)\left\|A u_{n}-A v\right\|^{2} \\
& \leq\left\|x_{n}-v\right\|^{2}+\lambda_{n}\left(\lambda_{n}-2 \alpha\right)\left\|A u_{n}-A v\right\|^{2}
\end{aligned}
$$

for every $n=1,2, \cdots$. Hence we have

$$
\begin{aligned}
& \left\|x_{n+1}-v\right\|^{2} \\
= & \left\|\alpha_{n}\left(f\left(x_{n}\right)-v\right)+\beta_{n}\left(x_{n}-v\right)+\gamma_{n}\left(z_{n}-v\right)\right\|^{2} \\
\leq \quad & \alpha_{n}^{2}\left\|f\left(x_{n}\right)-v\right\|^{2}+\beta_{n}^{2}\left\|x_{n}-v\right\|^{2}+\gamma_{n}^{2}\left\|\mu\left(S u_{n}-v\right)+(1-\mu)\left(t_{n}-v\right)\right\|^{2} \\
& +2 \alpha_{n} \beta_{n}\left\langle f\left(x_{n}\right)-v, x_{n}-v\right\rangle+2 \alpha_{n} \gamma_{n}\left\langle f\left(x_{n}\right)-v, z_{n}-v\right\rangle+2 \beta_{n} \gamma_{n}\left\|x_{n}-v\right\|^{2} \\
\leq \quad & \left.\alpha_{n}^{2}\left\|f\left(x_{n}\right)-v\right\|^{2}+\beta_{n}^{2}\left\|x_{n}-v\right\|^{2}+\gamma_{n}^{2} \mu\left\|S u_{n}-v\right\|^{2}+(1-\mu) \gamma_{n}^{2} \| t_{n}-v\right) \|^{2} \\
& +2 \alpha_{n} \beta_{n}\left\langle f\left(x_{n}\right)-v, x_{n}-v\right\rangle+2 \alpha_{n} \gamma_{n}\left\langle f\left(x_{n}\right)-v, z_{n}-v\right\rangle+2 \beta_{n} \gamma_{n}\left\|x_{n}-v\right\|^{2} \\
\leq \quad & \alpha_{n}^{2}\left\|f\left(x_{n}\right)-v\right\|^{2}+\beta_{n}^{2}\left\|x_{n}-v\right\|^{2}+\gamma_{n}^{2} \mu\left\|x_{n}-v\right\|^{2}+(1-\mu) \gamma_{n}^{2}\left(\left\|x_{n}-v\right\|^{2}\right. \\
& \left.+\lambda_{n}\left(\lambda_{n}-2 \alpha\right)\left\|A u_{n}-A v\right\|^{2}\right)+2 \beta_{n} \gamma_{n}\left\|x_{n}-v\right\|^{2}+2 \alpha_{n} \beta_{n}\left\langle f\left(x_{n}\right)-v, x_{n}-v\right\rangle \\
& +2 \alpha_{n} \gamma_{n}\left\langle f\left(x_{n}\right)-v, z_{n}-v\right\rangle \\
= & \alpha_{n}^{2}\left\|f\left(x_{n}\right)-v\right\|^{2}+\left(1-\alpha_{n}\right)^{2}\left\|x_{n}-v\right\|^{2}+(1-\mu) \gamma_{n}^{2} \lambda_{n}\left(\lambda_{n}-2 \alpha\right)\left\|A u_{n}-A v\right\|^{2} \\
& +2 \alpha_{n} \beta_{n}\left\langle f\left(x_{n}\right)-v, x_{n}-v\right\rangle+2 \alpha_{n} \gamma_{n}\left\langle f\left(x_{n}\right)-v, z_{n}-v\right\rangle \\
\leq \quad & \alpha_{n}^{2}\left\|f\left(x_{n}\right)-v\right\|^{2}+\left\|x_{n}-v\right\|^{2}+(1-\mu) \gamma_{n}^{2} a(b-2 \alpha)\left\|A u_{n}-A v\right\|^{2} \\
& +2 \alpha_{n} \beta_{n}\left\langle f\left(x_{n}\right)-v, x_{n}-v\right\rangle+2 \alpha_{n} \gamma_{n}\left\langle f\left(x_{n}\right)-v, z_{n}-v\right\rangle, \\
&
\end{aligned}
$$

i.e.,

$$
\begin{aligned}
& (1-\mu) \gamma_{n}^{2} a(2 \alpha-b)\left\|A u_{n}-A v\right\|^{2} \\
\leq \quad & \alpha_{n}^{2}\left\|f\left(x_{n}\right)-v\right\|^{2}+\left(\left\|x_{n+1}-v\right\|+\left\|x_{n}-v\right\|\right)\left(\left\|x_{n+1}-x_{n}\right\|\right) \\
& +2 \alpha_{n} \beta_{n}\left\langle f\left(x_{n}\right)-v, x_{n}-v\right\rangle+2 \alpha_{n} \gamma_{n}\left\langle f\left(x_{n}\right)-v, z_{n}-v\right\rangle,
\end{aligned}
$$


for every $n=1,2, \cdots$.

Noting $\lim _{n \rightarrow \infty} \alpha_{n}=0$, and $\left\{x_{n}\right\},\left\{f\left(x_{n}\right)\right\},\left\{z_{n}\right\}$ are bounded, by (3.4) we have

$$
\lim _{n \rightarrow \infty}\left\|A u_{n}-A v\right\|=0 .
$$

For every $n=1,2, \cdots$, by computing

$$
\begin{aligned}
\left\|t_{n}-v\right\|^{2}= & \left\|P_{C}\left(u_{n}-\lambda_{n} A u_{n}\right)-P_{C}\left(v-\lambda_{n} A v\right)\right\|^{2} \\
\leq & \left\langle\left(u_{n}-\lambda_{n} A u_{n}\right)-\left(v-\lambda_{n} A v\right), t_{n}-v\right\rangle \\
= & \frac{1}{2}\left\{\left\|\left(u_{n}-\lambda_{n} A u_{n}\right)-\left(v-\lambda_{n} A v\right)\right\|^{2}+\left\|t_{n}-v\right\|^{2}\right. \\
& \left.-\left\|\left(u_{n}-\lambda_{n} A u_{n}\right)-\left(v-\lambda_{n} A v\right)-\left(t_{n}-v\right)\right\|^{2}\right\} \\
\leq & \frac{1}{2}\left\{\left\|u_{n}-v\right\|^{2}+\left\|t_{n}-v\right\|^{2}-\left\|\left(u_{n}-t_{n}\right)-\lambda_{n}\left(A u_{n}-A v\right)\right\|^{2}\right\} \\
= & \frac{1}{2}\left\{\left\|u_{n}-v\right\|^{2}+\left\|t_{n}-v\right\|^{2}-\left\|u_{n}-t_{n}\right\|^{2}\right. \\
& \left.+2 \lambda_{n}\left\langle y u_{n}-t_{n}, A u_{n}-A v\right\rangle-\lambda_{n}^{2}\left\|A u_{n}-A v\right\|^{2}\right\}
\end{aligned}
$$

we obtain

$$
\begin{aligned}
& \left\|t_{n}-v\right\|^{2} \\
\leq & \left\|u_{n}-u\right\|^{2}-\left\|u_{n}-t_{n}\right\|^{2}+2 \lambda_{n}\left\langle u_{n}-t_{n}, A u_{n}-A v\right\rangle-\lambda_{n}^{2}\left\|A u_{n}-A v\right\|^{2} \\
\leq & \left\|x_{n}-u\right\|^{2}-\left\|u_{n}-t_{n}\right\|^{2}+2 \lambda_{n}\left\langle u_{n}-t_{n}, A u_{n}-A v\right\rangle-\lambda_{n}^{2}\left\|A u_{n}-A v\right\|^{2} .
\end{aligned}
$$

Therefore we have

$$
\begin{aligned}
& \left\|x_{n+1}-v\right\|^{2} \\
= & \left\|\alpha_{n}\left(f\left(x_{n}\right)-v\right)+\beta_{n}\left(x_{n}-v\right)+\gamma_{n}\left(z_{n}-v\right)\right\|^{2} \\
\leq & \alpha_{n}^{2}\left\|f\left(x_{n}\right)-v\right\|^{2}+\beta_{n}^{2}\left\|x_{n}-v\right\|^{2}+\gamma_{n}^{2}\left\|\mu\left(S u_{n}-v\right)+(1-\mu)\left(t_{n}-v\right)\right\|^{2} \\
& +2 \alpha_{n} \beta_{n}\left\langle f\left(x_{n}\right)-v, x_{n}-v\right\rangle+2 \alpha_{n} \gamma_{n}\left\langle f\left(x_{n}\right)-v, z_{n}-v\right\rangle+2 \beta_{n} \gamma_{n}\left\|x_{n}-v\right\|^{2} \\
\leq & \left.\alpha_{n}^{2}\left\|f\left(x_{n}\right)-v\right\|^{2}+\beta_{n}^{2}\left\|x_{n}-v\right\|^{2}+\gamma_{n}^{2} \mu\left\|x_{n}-v\right\|^{2}+(1-\mu) \gamma_{n}^{2} \| t_{n}-v\right) \|^{2} \\
& +2 \alpha_{n} \beta_{n}\left\langle f\left(x_{n}\right)-v, x_{n}-v\right\rangle+2 \alpha_{n} \gamma_{n}\left\langle f\left(x_{n}\right)-v, z_{n}-v\right\rangle+2 \beta_{n} \gamma_{n}\left\|x_{n}-v\right\|^{2} \\
\leq & \alpha_{n}^{2}\left\|f\left(x_{n}\right)-v\right\|^{2}+\beta_{n}^{2}\left\|x_{n}-v\right\|^{2}+\gamma_{n}^{2} \mu\left\|x_{n}-v\right\|^{2}+(1-\mu) \gamma_{n}^{2}\left(\left\|x_{n}-u\right\|^{2}\right. \\
& \left.-\left\|u_{n}-t_{n}\right\|^{2}+2 \lambda_{n}\left\langle u_{n}-t_{n}, A u_{n}-A v\right\rangle-\lambda_{n}^{2}\left\|A u_{n}-A v\right\|^{2}\right) \\
& +2 \alpha_{n} \beta_{n}\left\langle f\left(x_{n}\right)-v, x_{n}-v\right\rangle+2 \alpha_{n} \gamma_{n}\left\langle f\left(x_{n}\right)-v, z_{n}-v\right\rangle+2 \beta_{n} \gamma_{n}\left\|x_{n}-v\right\|^{2} \\
= & \alpha_{n}^{2}\left\|f\left(x_{n}\right)-v\right\|^{2}+\left(1-\alpha_{n}\right)^{2}\left\|x_{n}-v\right\|^{2}-(1-\mu) \gamma_{n}^{2}\left\|u_{n}-t_{n}\right\|^{2} \\
& +2 \alpha_{n} \beta_{n}\left\langle f\left(x_{n}\right)-v, x_{n}-v\right\rangle+2 \alpha_{n} \gamma_{n}\left\langle f\left(x_{n}\right)-v, z_{n}-v\right\rangle \\
& +2(1-\mu) \gamma_{n}^{2}\left(\lambda_{n}\left\langle u_{n}-t_{n}, A u_{n}-A v\right\rangle-\lambda_{n}^{2}\left\|A u_{n}-A v\right\|^{2}\right) \\
\leq & \alpha_{n}^{2}\left\|f\left(x_{n}\right)-v\right\|^{2}+\left\|x_{n}-v\right\|^{2}-(1-\mu) \gamma_{n}^{2}\left\|u_{n}-t_{n}\right\|^{2} \\
& +2 \alpha_{n} \beta_{n}\left\langle f\left(x_{n}\right)-v, x_{n}-v\right\rangle+2 \alpha_{n} \gamma_{n}\left\langle f\left(x_{n}\right)-v, z_{n}-v\right\rangle \\
& +2(1-\mu) \gamma_{n}^{2}\left(\lambda_{n}\left\langle u_{n}-t_{n}, A u_{n}-A v\right\rangle-\lambda_{n}^{2}\left\|A u_{n}-A v\right\|^{2}\right), \\
&
\end{aligned}
$$

i.e.,

$$
\begin{aligned}
& (1-\mu) \gamma_{n}^{2}\left\|u_{n}-t_{n}\right\|^{2} \\
\leq & \alpha_{n}^{2}\left\|f\left(x_{n}\right)-v\right\|^{2}+\left(\left\|x_{n}-v\right\|+\left\|x_{n+1}-v\right\|\right)\left\|x_{n}-x_{n+1}\right\| \\
& +2 \alpha_{n} \beta_{n}\left\langle f\left(x_{n}\right)-v, x_{n}-v\right\rangle+2 \alpha_{n} \gamma_{n}\left\langle f\left(x_{n}\right)-v, z_{n}-v\right\rangle \\
& +2(1-\mu) \gamma_{n}^{2}\left(\lambda_{n}\left\langle u_{n}-t_{n}, A u_{n}-A v\right\rangle-\lambda_{n}^{2}\left\|A u_{n}-A v\right\|^{2}\right),
\end{aligned}
$$

for every $n=1,2, \cdots$. By using (2.4) and (2.5), then noting $\lim _{n \rightarrow \infty} \alpha_{n}=0$, we have

$$
\lim _{n \rightarrow \infty}\left\|u_{n}-t_{n}\right\|=0 .
$$

Next we prove that $\lim _{n \rightarrow \infty}\left\|x_{n}-u_{n}\right\|=0$. Since

$$
\begin{aligned}
\left\|u_{n}-v\right\|^{2} & =\left\|T_{r_{n}} x_{n}-T_{r_{n}} v\right\|^{2} \\
& \leq\left\langle T_{r_{n}} x_{n}-T_{r_{n}} v, x_{n}-v\right\rangle \\
& =\left\langle u_{n}-v, x_{n}-v\right\rangle \\
& =\frac{1}{2}\left(\left\|u_{n}-v\right\|^{2}+\left\|x_{n}-v\right\|^{2}-\left\|x_{n}-u_{n}\right\|^{2}\right),
\end{aligned}
$$


we have

$$
\left\|u_{n}-v\right\|^{2} \leq\left\|x_{n}-v\right\|^{2}-\left\|x_{n}-u_{n}\right\|^{2}
$$

for every $n=1,2, \cdots$. Therefore, we have

$$
\begin{aligned}
& \left\|x_{n+1}-v\right\|^{2} \\
= & \left\|\alpha_{n}\left(f\left(x_{n}\right)-v\right)+\beta_{n}\left(x_{n}-v\right)+\gamma_{n}\left(z_{n}-v\right)\right\|^{2} \\
\leq & \alpha_{n}^{2}\left\|f\left(x_{n}\right)-v\right\|^{2}+\beta_{n}^{2}\left\|x_{n}-v\right\|^{2}+\gamma_{n}^{2}\left\|\mu\left(S u_{n}-v\right)+(1-\mu)\left(t_{n}-v\right)\right\|^{2} \\
& +2 \alpha_{n} \beta_{n}\left\langle f\left(x_{n}\right)-v, x_{n}-v\right\rangle+2 \alpha_{n} \gamma_{n}\left\langle f\left(x_{n}\right)-v, z_{n}-v\right\rangle+2 \beta_{n} \gamma_{n}\left\|x_{n}-v\right\|^{2} \\
\leq \quad & \alpha_{n}^{2}\left\|f\left(x_{n}\right)-v\right\|^{2}+\beta_{n}^{2}\left\|x_{n}-v\right\|^{2}+\gamma_{n}^{2} \mu\left\|u_{n}-v\right\|^{2}+(1-\mu) \gamma_{n}^{2}\left\|t_{n}-v\right\|^{2} \\
& +2 \alpha_{n} \beta_{n}\left\langle f\left(x_{n}\right)-v, x_{n}-v\right\rangle+2 \alpha_{n} \gamma_{n}\left\langle f\left(x_{n}\right)-v, z_{n}-v\right\rangle+2 \beta_{n} \gamma_{n}\left\|x_{n}-v\right\|^{2} \\
\leq \quad & \alpha_{n}^{2}\left\|f\left(x_{n}\right)-v\right\|^{2}+\beta_{n}^{2}\left\|x_{n}-v\right\|^{2}+\gamma_{n}^{2} \mu\left(\left\|x_{n}-v\right\|^{2}-\left\|x_{n}-u_{n}\right\|^{2}\right) \\
& +(1-\mu) \gamma_{n}^{2}\left\|x_{n}-v\right\|^{2}+2 \alpha_{n} \beta_{n}\left\langle f\left(x_{n}\right)-v, x_{n}-v\right\rangle \\
& +2 \alpha_{n} \gamma_{n}\left\langle f\left(x_{n}\right)-v, z_{n}-v\right\rangle+2 \beta_{n} \gamma_{n}\left\|x_{n}-v\right\|^{2} \\
\leq \quad & \alpha_{n}^{2}\left\|f\left(x_{n}\right)-v\right\|^{2}+\left\|x_{n}-v\right\|^{2}-\gamma_{n}^{2} \mu\left\|x_{n}-u_{n}\right\|^{2}+2 \alpha_{n} \beta_{n}\left\langle f\left(x_{n}\right)-v, x_{n}-v\right\rangle \\
& +2 \alpha_{n} \gamma_{n}\left\langle f\left(x_{n}\right)-v, z_{n}-v\right\rangle,
\end{aligned}
$$

i.e.,

$$
\begin{aligned}
\gamma_{n}^{2} \mu\left\|x_{n}-u_{n}\right\|^{2} \leq & \alpha_{n}^{2}\left\|f\left(x_{n}\right)-v\right\|^{2}+\left(\left\|x_{n}-v\right\|+\left\|x_{n+1}-v\right\|\right)\left\|x_{n}-x_{n+1}\right\| \\
& +2 \alpha_{n} \beta_{n}\left\langle f\left(x_{n}\right)-v, x_{n}-v\right\rangle+2 \alpha_{n} \gamma_{n}\left\langle f\left(x_{n}\right)-v, z_{n}-v\right\rangle
\end{aligned}
$$

which implies that

$$
\lim _{n \rightarrow \infty}\left\|x_{n}-u_{n}\right\|=0
$$

Step 4. $\lim _{n \rightarrow \infty}\left\|x_{n}-S x_{n}\right\|=0$ and $\lim _{n \rightarrow \infty}\left\|x_{n}-P_{C}\left(x_{n}-\lambda A x_{n}\right)\right\|=0$, where $\lim _{n \rightarrow \infty} \lambda_{n}=\lambda \in(0,2 \alpha)$.

Since $\left\|x_{n}-t_{n}\right\| \leq\left\|x_{n}-u_{n}\right\|+\left\|u_{n}-t_{n}\right\|$, by using (2.5) and (2.6) we have

$$
\lim _{n \rightarrow \infty}\left\|x_{n}-t_{n}\right\|=0
$$

However, since $x_{n+1}-x_{n}=\alpha_{n}\left(f\left(x_{n}\right)-x_{n}\right)+\gamma_{n} \mu\left(S u_{n}-x_{n}\right)+\gamma_{n}(1-\mu)\left(t_{n}-x_{n}\right)$, we have

$$
\gamma_{n} \mu\left\|S u_{n}-x_{n}\right\| \leq\left\|x_{n+1}-x_{n}\right\|+\alpha_{n}\left(\left\|f\left(x_{n}\right)\right\|+\left\|x_{n}\right\|\right)+\left\|t_{n}-x_{n}\right\|,
$$

for every $n=1,2, \cdots$. Noting $\left\{f\left(x_{n}\right)\right\}$ and $\left\{x_{n}\right\}$ are bounded, $\lim _{n \rightarrow \infty} \alpha_{n}=0$, then by (2.4) and (2.8) we obtain

$$
\lim _{n \rightarrow \infty}\left\|x_{n}-S u_{n}\right\|=0 .
$$

Since $\left\|u_{n}-S u_{n}\right\| \leq\left\|u_{n}-x_{n}\right\|+\left\|x_{n}-S u_{n}\right\|$, for every $n=1,2, \cdots$, from (2.7) and (2.9) we obtain

$$
\lim _{n \rightarrow \infty}\left\|u_{n}-S u_{n}\right\|=0
$$

Noting

$$
\begin{aligned}
\left\|x_{n}-S x_{n}\right\| & \leq\left\|x_{n}-S u_{n}\right\|+\left\|S u_{n}-S x_{n}\right\| \\
& \leq\left\|x_{n}-S u_{n}\right\|+\left\|u_{n}-x_{n}\right\|,
\end{aligned}
$$


for every $n=1,2, \cdots$, by $(2.7)$ and (2.9) we have

$$
\lim _{n \rightarrow \infty}\left\|x_{n}-S x_{n}\right\|=0 .
$$

Next we prove that $\lim _{n \rightarrow \infty}\left\|x_{n}-P_{C}\left(x_{n}-\lambda A x_{n}\right)\right\|=0$. To see this, putting $y_{n}=P_{C}\left(x_{n}-\lambda_{n} A x_{n}\right)$, for every $n=1,2, \cdots$, we have

$$
\begin{aligned}
\left\|x_{n}-y_{n}\right\| & \leq\left\|x_{n}-t_{n}\right\|+\left\|t_{n}-y_{n}\right\| \\
& =\left\|x_{n}-t_{n}\right\|+\left\|P_{C}\left(u_{n}-\lambda_{n} A u_{n}\right)-P_{C}\left(x_{n}-\lambda_{n} A x_{n}\right)\right\| \\
& \leq\left\|x_{n}-t_{n}\right\|+\left\|u_{n}-\lambda_{n} A u_{n}-\left(x_{n}-\lambda_{n} A x_{n}\right)\right\| \\
& \leq\left\|x_{n}-t_{n}\right\|+\left\|u_{n}-x_{n}\right\|,
\end{aligned}
$$

by (2.7) and (2.8) we obtain

$$
\lim _{n \rightarrow \infty}\left\|x_{n}-y_{n}\right\|=0
$$

Hence,

$$
\lim _{n \rightarrow \infty}\left\|x_{n}-P_{C}\left(x_{n}-\lambda A x_{n}\right)\right\|=\lim _{n \rightarrow \infty}\left\|x_{n}-P_{C}\left(x_{n}-\lambda_{n} A x_{n}\right)\right\|=0 .
$$

Step 5. $\lim _{\sup _{n \rightarrow \infty}}\left\langle f(z)-z, x_{n}-z\right\rangle \leq 0$, where $z=P_{F(S) \cap E P(F) \cap V I(C, A)} f(z)$.

Since $\left\{x_{n}\right\}$ is bounded, we may choose a subsequence $\left\{x_{n_{i}}\right\}$ of $\left\{x_{n}\right\}$ such that

$$
\limsup _{n \rightarrow \infty}\left\langle f(z)-z, x_{n}-z\right\rangle=\lim _{i \rightarrow \infty}\left\langle f(z)-z, x_{n_{i}}-z\right\rangle .
$$

As $\left\{x_{n_{i}}\right\}$ is also bounded, we can choose a subsequence $\left\{x_{n_{i_{j}}}\right\}$ of $\left\{x_{n_{i}}\right\}$ converges weakly to $p$. Without loss of generality we may assume that $x_{n_{i}} \rightarrow p$, then we have $p \in F(S) \cap E P(F) \cap V I(C, A)$.

First we show $p \in F(S) \cap V I(C, A)$. Since $x_{n}-S x_{n} \rightarrow 0$ and $x_{n}-P_{C}\left(x_{n}-\right.$ $\left.\lambda A x_{n}\right) \rightarrow 0$, by the demiclosedness principle for nonexpansive mappings, we obtain $p=S p$ and $p=P_{C}(p-\lambda A p)$, i.e., $p \in F(S) \cap V I(C, A)$.

Next we show that $p \in E P(F)$. In fact, since $\lim _{i \rightarrow \infty}\left\|x_{n_{i}}-u_{n_{i}}\right\|=0$, we have $\left\{u_{n_{i}}\right\}$ also converges weakly to $p$. From $\left\|S u_{n}-u_{n}\right\| \rightarrow 0$, we obtain $S u_{n_{i}} \rightarrow p$. By $u_{n}=T_{r_{n}} x_{n}$, we have

$$
F\left(u_{n}, y\right)+\frac{1}{r_{n}}\left\langle y-u_{n}, u_{n}-x_{n}\right\rangle \geq 0, \quad \forall y \in C .
$$

From (A2), we also have

$$
\frac{1}{r_{n}}\left\langle y-u_{n}, u_{n}-x_{n}\right\rangle \geq F\left(y, u_{n}\right)
$$

and hence

$$
\left\langle y-u_{n_{i}}, \frac{u_{n_{i}}-x_{n_{i}}}{r_{n_{i}}}\right\rangle \geq F\left(y, u_{n_{i}}\right) .
$$

Since $\liminf \operatorname{in}_{n \rightarrow \infty} r_{n}>0, \frac{u_{n_{i}}-x_{n_{i}}}{r_{n_{i}}} \rightarrow 0$ and $u_{n_{i}} \rightarrow p$, from (A4) we have

$$
F(y, p) \leq 0
$$


for all $y \in C$. For $t$ with $0<t \leq 1$ and $y \in C$, let $y_{t}=t y+(1-t) p$. Since $y \in C$ and $p \in C$, we have $y_{t} \in C$ and hence $F\left(y_{t}, p\right) \leq 0$. Therefore, from (A1) and (A4) we have

$$
\begin{aligned}
F\left(y_{t}, y_{t}\right) & \leq t F\left(y_{t}, y\right)+(1-t) F\left(y_{t}, p\right) \\
& \leq t F\left(y_{t}, y\right) .
\end{aligned}
$$

Noting $F\left(y_{t}, y_{t}\right)=0$ and $0<t \leq 1$, we have $0 \leq F\left(y_{t}, y\right)$. From (A3), we have $0 \leq F(p, y)$ for all $y \in C$, which implies that $p \in E P(F)$. Therefore, $p \in F(S) \cap$ $E P(F) \cap V I(C, A)$. Since $z=P_{F(S) \cap E P(F) \cap V I(C, A)} f(z)$, we have

$$
\begin{aligned}
\lim \sup _{n \rightarrow \infty}\left\langle f(z)-z, x_{n}-z\right\rangle & =\lim _{i \rightarrow \infty}\left\langle f(z)-z, x_{n_{i}}-z\right\rangle \\
& =\langle f(z)-z, p-z\rangle \leq 0 .
\end{aligned}
$$

Since $x_{n}-S u_{n} \rightarrow 0$ and $x_{n}-t_{n} \rightarrow 0$, we have $x_{n}-z_{n} \rightarrow 0$. Hence, we have

$$
\limsup _{n \rightarrow \infty}\left\langle f(z)-z, z_{n}-z\right\rangle \leq 0 \text {. }
$$

Step 6. $\lim _{n \rightarrow \infty}\left\|x_{n}-z\right\|=0$.

For $z=P_{F(S) \cap E P(F) \cap V I(C, A)} f(z)$, we have

$$
\begin{aligned}
& \left\|x_{n+1}-z\right\|^{2} \\
= & \left\|\alpha_{n}\left(f\left(x_{n}\right)-z\right)+\beta_{n}\left(x_{n}-z\right)+\gamma_{n}\left(z_{n}-z\right)\right\|^{2} \\
= & \alpha_{n}^{2}\left\|f\left(x_{n}\right)-z\right\|^{2}+\beta_{n}^{2}\left\|x_{n}-z\right\|^{2}+\gamma_{n}^{2}\left\|z_{n}-z\right\|^{2}+2 \alpha_{n} \beta_{n}\left\langle f\left(x_{n}\right)-z, x_{n}-z\right\rangle \\
& 2 \alpha_{n} \gamma_{n}\left\langle f\left(x_{n}\right)-z, z_{n}-z\right\rangle+2 \beta_{n} \gamma_{n}\left\langle x_{n}-z, z_{n}-z\right\rangle \\
\leq & \alpha_{n}^{2}\left\|f\left(x_{n}\right)-z\right\|^{2}+\beta_{n}^{2}\left\|x_{n}-z\right\|^{2}+\gamma_{n}^{2}\left\|x_{n}-z\right\|^{2}+2 \beta_{n} \gamma_{n}\left\|x_{n}-z\right\|^{2} \\
& +2 \alpha_{n} \beta_{n} \kappa\left\|x_{n}-z\right\|^{2}+2 \alpha_{n} \beta_{n}\left\langle f(z)-z, x_{n}-z\right\rangle+2 \alpha_{n} \gamma_{n} \kappa\left\|x_{n}-z\right\|^{2} \\
& +2 \alpha_{n} \gamma_{n}\left\langle f(z)-z, z_{n}-z\right\rangle \\
= & \left(1-2 \alpha_{n}+\alpha_{n}^{2}+2 \kappa \alpha_{n}\left(1-\alpha_{n}\right)\right)\left\|x_{n}-z\right\|^{2}+\alpha_{n}^{2}\left\|f\left(x_{n}\right)-z\right\|^{2} \\
& +2 \alpha_{n} \beta_{n}\left\langle f(z)-z, x_{n}-z\right\rangle+2 \alpha_{n} \gamma_{n}\left\langle f(z)-z, z_{n}-z\right\rangle,
\end{aligned}
$$

for every $n=1,2, \cdots$. Put $\sigma_{n}^{1}=\max \left\{0,\left\langle f(z)-z, x_{n}-z\right\rangle\right\}$ and $\sigma_{n}^{2}=\max \{0,\langle f(z)-$ $\left.\left.z, z_{n}-z\right\rangle\right\}$, then $\sigma_{n}^{1} \geq 0$ and $\sigma_{n}^{2} \geq 0$, for every $n=1,2, \cdots$. Hence, we have

$$
\left\|x_{n+1}-z\right\|^{2} \leq\left(1-\bar{\alpha}_{n}\right)\left\|x_{n}-z\right\|^{2}+\alpha_{n}^{2}\left\|f\left(x_{n}\right)-z\right\|^{2}+2 \alpha_{n} \beta_{n} \sigma_{n}^{1}+2 \beta_{n} \gamma_{n} \sigma_{n}^{2},
$$

where $\bar{\alpha}_{n}=\alpha_{n}\left(2-\alpha_{n}-2 \kappa\left(1-\alpha_{n}\right)\right)$. From (2.10) and (2.11), we have

$$
\sigma_{n}^{1} \rightarrow 0 \quad \text { and } \quad \sigma_{n}^{2} \rightarrow 0
$$

Therefore, we have

$$
\left\|x_{n+1}-z\right\|^{2} \leq\left(1-\bar{\alpha}_{n}\right)\left\|x_{n}-z\right\|^{2}+o\left(\bar{\alpha}_{n}\right) .
$$

Since $\lim _{n \rightarrow \infty} \bar{\alpha}_{n}=0$ and $\sum_{n=1}^{\infty} \bar{\alpha}_{n}=\infty$, by Lemma 1.4 we have

$$
\lim _{n \rightarrow \infty}\left\|x_{n}-z\right\|=0 \text {. }
$$

This theorem is complete.

As direct consequences of Theorem 2.1, we obtain two corollaries. 
Corollary 2.2. Let $C$ be a nonempty closed convex subset of a real Hilbert space $H$. Let $f$ be a contraction from $C$ into itself. Let $A$ be an $\alpha$-inverse-strongly monotone mapping of $C$ into $H$ and $S$ be a nonexpansive mapping of $C$ into itself such that $F(S) \cap V I(C, A) \neq \emptyset$. Suppose $x_{1}=x \in C$ and $\left\{x_{n}\right\}$ is a sequence generated by

$$
x_{n+1}=\alpha_{n} f\left(x_{n}\right)+\beta_{n} x_{n}+\gamma_{n}\left(\mu S x_{n}+(1-\mu) P_{C}\left(x_{n}-\lambda_{n} A x_{n}\right)\right),
$$

for every $n=1,2, \cdots$, where $\mu \in[0,1],\left\{\lambda_{n}\right\} \subset[a, b]$ with $0<a<b<2 \alpha$ and $\left\{\alpha_{n}\right\},\left\{\beta_{n}\right\}$ and $\left\{\gamma_{n}\right\}$ are sequences in $[0,1]$ and satisfy $\alpha_{n}+\beta_{n}+\gamma_{n}=1$ for every $n=1,2, \cdots$. If $\left\{\alpha_{n}\right\},\left\{\beta_{n}\right\}$ and $\left\{\lambda_{n}\right\}$ are chosen so that

$\lim _{n \rightarrow \infty} \alpha_{n}=0, \sum_{n=1}^{\infty} \alpha_{n}=\infty, \quad 0<\liminf _{n \rightarrow \infty} \beta_{n}<\limsup _{n \rightarrow \infty} \beta_{n}<1$ and $\lim _{n \rightarrow \infty}\left|\lambda_{n+1}-\lambda_{n}\right|=0$,

then $\left\{x_{n}\right\}$ converges strongly to $z \in F(S) \cap V I(C, A)$, where $z=P_{F(S) \cap V I(C, A)} f(z)$.

Proof. Put $F(x, y)=0$ for all $x, y \in C$ and $r_{n}=1$ for all $n=1,2, \cdots$ in Theorem 2.1. Then, we have $u_{n}=P_{C} x_{n}=x_{n}$. So, from Theorem 2.1, the sequence $\left\{x_{n}\right\}$ in Corollary 2.2 converges strongly to $z \in F(S) \cap V I(C, A)$, where $z=P_{F(S) \cap V I(C, A)} f(z)$.

Corollary 2.3. Let $C$ be a nonempty closed convex subset of a real Hilbert space $H$. Let $F$ be a bifunction from $C \times C$ to $\mathbf{R}$ satisfying $(\mathrm{A} 1)-(\mathrm{A} 4)$ and $f$ be a contraction from $C$ into itself. Let $A$ be an $\alpha$-inverse-strongly monotone mapping of $C$ into $H$ such that $\operatorname{EP}(F) \cap V I(C, A) \neq \emptyset$. Suppose $x_{1}=x \in C$ and $\left\{x_{n}\right\},\left\{u_{n}\right\}$ are sequences generated by

$$
\left\{\begin{array}{l}
F\left(u_{n}, y\right)+\frac{1}{r_{n}}\left\langle y-u_{n}, u_{n}-x_{n}\right\rangle \geq 0, \quad \forall y \in C \\
x_{n+1}=\alpha_{n} f\left(x_{n}\right)+\beta_{n} x_{n}+\gamma_{n}\left(\mu u_{n}+(1-\mu) P_{C}\left(u_{n}-\lambda_{n} A u_{n}\right)\right),
\end{array}\right.
$$

for every $n=1,2, \cdots$, where $\mu \in[0,1],\left\{r_{n}\right\} \subset(0, \infty),\left\{\lambda_{n}\right\} \subset[a, b]$ with $0<a<b<$ $2 \alpha$ and $\left\{\alpha_{n}\right\},\left\{\beta_{n}\right\}$ and $\left\{\gamma_{n}\right\}$ are sequences in $[0,1]$ and satisfy $\alpha_{n}+\beta_{n}+\gamma_{n}=1$ for every $n=1,2, \cdots$. If $\left\{\alpha_{n}\right\},\left\{\beta_{n}\right\},\left\{\lambda_{n}\right\}$ and $\left\{r_{n}\right\}$ are chosen so that

$$
\begin{aligned}
& \lim _{n \rightarrow \infty} \alpha_{n}=0, \quad \sum_{n=1}^{\infty} \alpha_{n}=\infty, \quad 0<\liminf _{n \rightarrow \infty} \beta_{n}<\limsup _{n \rightarrow \infty} \beta_{n}<1, \\
& \lim _{n \rightarrow \infty}\left|\lambda_{n+1}-\lambda_{n}\right|=0, \quad \liminf _{n \rightarrow \infty} r_{n}>0 \text { and } \lim _{n \rightarrow \infty}\left|r_{n+1}-r_{n}\right|=0,
\end{aligned}
$$

then $\left\{x_{n}\right\}$ and $u_{n}$ converge strongly to $z \in \operatorname{EP}(F) \cap V I(C, A)$, where $z=$ $P_{E P(F) \cap V I(C, A)} f(z)$.

Proof. This conclusion may be directly obtained by putting $S x=x$ for all $x \in C$ in Theorem 2.1.

REMARK 2.4. We may obtain Wittmann's theorem [12] if $f(y)=x_{1}$ for all $y \in C, \beta_{n} \equiv 0$ and $\mu=1$ in Corollary 2.2. We also obtain Combettes and Hirstoaga's theorem [5] if $f$ is a contraction from $H$ into itself and $f(y)=x_{1}=x \in H$ for all $y \in H, \mu=1$ and $\beta_{n} \equiv 1$ in Corollary 2.3. 
3. Applications. In this section, we first prove one theorem in a real Hilbert space $H$ by using Theorem 2.1 .

Let $C$ be a closed convex subset of $H$, and $T$ be a mapping from $C$ to $C$. $T$ is called strictly pseudo-contractive if there exists some $\kappa$ with $0 \leq \kappa<1$ such that

$$
\|T x-T y\|^{2} \leq\|x-y\|^{2}+\kappa\|(I-T) x-(I-T) y\|^{2}
$$

for all $x, y \in C$.

Put $A=I-T$, where $T: C \rightarrow C$ is a $\kappa$-strictly pseudo-contractive mapping. Then $A$ is a $\frac{1-\kappa}{2}$-inverse-strongly monotone mapping [3].

Theorem 3.1. Let $C$ be a closed convex subset of a real Hilbert space H. Let $F$ be a bifunction from $C \times C$ to $\mathbf{R}$ satisfying (A1) - (A4) and $f$ be a contraction from $C$ into itself. Let $S$ be an nonexpansive mapping of $C$ into itself and $T$ be a $\kappa$-strictly pseudo-contractive mapping of $C$ into itself such that $F(S) \cap E P(F) \cap F(T) \neq \emptyset$. Suppose $x_{1}=x \in C$ and $\left\{x_{n}\right\},\left\{u_{n}\right\}$ are given by

$$
\left\{\begin{array}{l}
F\left(u_{n}, y\right)+\frac{1}{r_{n}}\left\langle y-u_{n}, u_{n}-x_{n}\right\rangle \geq 0, \quad \forall y \in C, \\
x_{n+1}=\alpha_{n} f\left(x_{n}\right)+\beta_{n} x_{n}+\gamma_{n}\left(\mu S u_{n}+(1-\mu)\left(\left(1-\lambda_{n}\right) u_{n}-\lambda_{n} T u_{n}\right)\right)
\end{array}\right.
$$

for every $n=1,2, \cdots$, where $\mu \in[0,1],\left\{r_{n}\right\} \subset(0, \infty),\left\{\lambda_{n}\right\} \subset[a, b]$ with $0<a<b<$ $1-\kappa$ and $\left\{\alpha_{n}\right\},\left\{\beta_{n}\right\}$ and $\left\{\gamma_{n}\right\}$ are sequences in $[0,1]$ and satisfy $\alpha_{n}+\beta_{n}+\gamma_{n}=1$ for every $n=1,2, \cdots$. If $\left\{\alpha_{n}\right\},\left\{\beta_{n}\right\},\left\{\lambda_{n}\right\}$ and $\left\{r_{n}\right\}$ are chosen so that

$$
\begin{aligned}
& \lim _{n \rightarrow \infty} \alpha_{n}=0, \quad \sum_{n=1}^{\infty} \alpha_{n}=\infty, \quad 0<\liminf _{n \rightarrow \infty} \beta_{n}<\limsup _{n \rightarrow \infty} \beta_{n}<1, \\
& \lim _{n \rightarrow \infty}\left|\lambda_{n+1}-\lambda_{n}\right|=0, \quad \liminf _{n \rightarrow \infty} r_{n}>0 \text { and } \lim _{n \rightarrow \infty}\left|r_{n+1}-r_{n}\right|=0,
\end{aligned}
$$

then $\left\{x_{n}\right\}$ and $\left\{u_{n}\right\}$ converge strongly to $z \in F(S) \cap E P(F) \cap F(T)$, where $z=$ $P_{F(S) \cap E P(F) \cap F(T)} f(z)$.

Proof. Put $A=I-T$. Then $A$ is a $\frac{1-\kappa}{2}$-inverse-strongly monotone mapping. We have $F(T)=V I(C, A)$ and $P_{C}\left(u_{n}-\lambda_{n} A u_{n}\right)=\left(1-\lambda_{n}\right) u_{n}+\lambda_{n} T u_{n}$. So, by Theorem 2.1 , we obtain the desired result.

Next we consider the problem of finding a minimizer of a continuously Fréchet differentiable convex function in a Hilbert space $H$. Let $g$ be a continuously Fréchet differentiable convex function on $H$ and let $\nabla g$ be the gradient of $g$. It is known that if $\nabla g$ is $1 / \alpha$-Lipschitz continuous, then $\nabla g$ is $\alpha$-inverse-strongly monotone [1]. Moreover, we also obtain from the convexity and Fréchet differentiability of $g$ that

$$
V I(H, \nabla g)=(\nabla g)^{-1}(0),
$$

where $(\nabla g)^{-1}(0)=\left\{x \in H: g(x)=\min _{y \in H} g(y)\right\}$. So, if letting $F \equiv 0$ and $A=(\nabla g)^{-1}(0)$ in Theorem 2.1, then the iterative scheme in Theorem 2.1 converges strongly to $z \in F(S) \cap(\nabla g)^{-1}(0)$, which is a solution of the unconstrained optimization problem for the convex function $g$. Based on this idea, we give the following theorem:

THEOREM 3.2. Let $H$ be a real Hilbert space and let $f$ be a contraction from $H$ into itself. Let $g$ be a continuously Fréchet differentiable convex function on $H$ and 
assume that $\nabla g$ is $1 / \alpha$-Lipschitz continuous. Let $S$ be a nonexpansive mapping of $H$ into itself such that $F(S) \cap(\nabla g)^{-1}(0) \neq \emptyset$. Suppose $x_{1}=x \in H$ and $\left\{x_{n}\right\}$ is a sequence generated by

$$
x_{n+1}=\alpha_{n} f\left(x_{n}\right)+\beta_{n} x_{n}+\gamma_{n}\left(\mu S x_{n}+(1-\mu)\left(x_{n}-\lambda_{n} \nabla g x_{n}\right)\right),
$$

for every $n=1,2, \cdots$, where $\mu \in[0,1],\left\{\lambda_{n}\right\} \subset[a, b]$ with $0<a<b<2 \alpha$ and $\left\{\alpha_{n}\right\},\left\{\beta_{n}\right\}$ and $\left\{\gamma_{n}\right\}$ are sequences in $[0,1]$ and satisfy $\alpha_{n}+\beta_{n}+\gamma_{n}=1$ for every $n=1,2, \cdots$. If $\left\{\alpha_{n}\right\},\left\{\beta_{n}\right\}$ and $\left\{\lambda_{n}\right\}$ are chosen so that

$\lim _{n \rightarrow \infty} \alpha_{n}=0, \quad \sum_{n=1}^{\infty} \alpha_{n}=\infty, \quad 0<\liminf _{n \rightarrow \infty} \beta_{n}<\limsup _{n \rightarrow \infty} \beta_{n}<1$ and $\lim _{n \rightarrow \infty}\left|\lambda_{n+1}-\lambda_{n}\right|=0$,

then $\left\{x_{n}\right\}$ converges strongly to $z \in F(S) \cap(\nabla g)^{-1}(0)$, where $z=P_{F(S) \cap(\nabla g)^{-1}(0)} f(z)$.

Proof. Put $F(x, y)=0$ for all $x, y \in C$ and $r_{n}=1$ for all $n=1,2, \cdots$ in Theorem 2.1. Then, noting that $\nabla g$ is $\alpha$-inverse-strong monotone and $(\nabla g)^{-1}(0)=V I(H, \nabla g)$, this conclusion may be directly obtained by Theorem 2.1 .

REMARK 3.3. If $g$ is just a convex and lower semicontinuous function defined on a nonempty closed convex subset $C$ of $H$, we can also obtain the optimal solution of $g$ by the result of this paper. Denote by $A$ the set of solutions of the optimization problem

$$
\left\{\begin{array}{l}
\min g(x) \\
x \in C
\end{array}\right.
$$

We define the bifunction $F$ by $F(x, y)=g(y)-g(x)$ and denote by $E P(F)$ the set of solutions of the following equilibrium problem, that is to find $x \in C$ such that

$$
F(x, y) \geq 0, \quad \forall y \in C .
$$

Obviously, $F(x, y)$ satisfies the conditions $\mathrm{A}(1)-\mathrm{A}(4)$ and $E P(F)=A$. Therefore, from Corollary 2.3 we know that the following iterative algorithm

$$
\left\{\begin{array}{l}
F\left(u_{n}, y\right)+\left\langle y-u_{n}, u_{n}-x_{n}\right\rangle \geq 0, \quad \forall y \in C \\
x_{n+1}=\alpha_{n} f\left(x_{n}\right)+\beta_{n} x_{n}+\gamma_{n} u_{n}
\end{array}\right.
$$

for any initial guess $x_{1} \in C$ and all $n \geq 1$, converges strongly to a solution $z=P_{A} f(z)$ of optimization problem (3.1), where $\left\{\alpha_{n}\right\},\left\{\beta_{n}\right\}$ and $\left\{\gamma_{n}\right\}$ satisfy the conditions in Corollary 2.3.

Acknowledgments. The authors thank the referee for his or her comments and suggestions which improved the presentation of this manuscript. Haiyun Zhou was supported by the National Research Foundation of China (No. 10771050). Shenghua Wang was supported by Youth Foundation of North China Electric Power University.

\section{REFERENCES}

[1] J.B. BAILlON AND G. HADDAD, Quelques propriétés des opérateurs angle-bornés et $n$-cycliquement monotones, Israel J. Math., 26 (1977), pp. 137-150.

[2] E. Blum ABD W. OetTli, From optimization and variatinal inequalities to equilibrium problems, Math. Student, 63 (1994), pp. 123-145. 
[3] F.E. Browder And W.V. Petryshyn, Construction of fixed points of nonlinear mappings in Hiblert space, J. Math. Anal. Appl., 20 (1967), pp. 197-228.

[4] J.M. Chen, L.J. ZHANG AND T.G FAN, Viscosity approximation methods for nonexpansive mappings and monotone mappings, J. Math. Anal. Appl., 334 (2007), pp. 1450-1461.

[5] P.L. Combettes and S.A. Hirstoaga, Equilibrium programming in Hiblert spaces, J. Nonlinear Convex Anal., 6 (2005), pp. 117-136.

[6] H. IIDUKA AND W. TAKAHASHI, Strong convergence theorems for nonexpansive mappings and inverse-strongly monotone mappings, Nonlinear Anal., 61 (2005), pp. 341-350.

[7] X.L. QIn, M.J. Shang AND Y.F. Su, A general iterative method for equilibrium problems and fixed point problems in Hilbert spaces, Nonlinear Analysis (2007), doi:10.1016./j.na.2007.10.025.

[8] T. SuzukI, Strong convergence of Krasnoselskii and Mann's type sequences for one-parameter nonexpansive semi-groups without Bochner integrals, J. Math. Anal. Appl., 305 (2005), pp. $227-239$.

[9] S. TAKAHASHI AND W. TAKAHASHI, Viscosity approximation methods for equilibrium problems and fixed point problems in Hilbert spaces, J. Math. Anal. Appl., 331 (2007), pp. 506-515.

[10] S. TAKAHASHi AND W. TAKAHASHI, Strong convergence theorem for a generalized equilibrium problem and a nonexpansive mapping in a Hilbert space, Nonlinear Anal., 69 (2008), pp. 1025-1033.

[11] X. L. Weng, Fixed point iteration for local strictly pseudocontractive mappings, Proc. Amer. Math. Soc., 113 (1991), pp. 727-731.

[12] R. Wittmann, Approximation of fixed points of nonexpansive mappings, Arch. Math., 58 (1992), pp. 486-491. 
S. WANG, H. ZHOU AND J. SONG 